American Journal of Neuroscience 2 (1): 5-16, 2011

ISSN 1948-9900

C 2011 Science Publications

\title{
Effects of Repetitive Transcranial Magnetic Stimulation on Dystonia: An Overview
}

\author{
${ }^{1,2,3,4}$ Sergio Machado, ${ }^{5}$ Oscar Arias-Carrion, ${ }^{1,2,6}$ Flávia Paes, ${ }^{8}$ Emmy Uehara, \\ ${ }^{3,4}$ Bruna Velasques, ${ }^{4,9}$ Silmar Teixeira, ${ }^{9}$ Andre dos Santos, ${ }^{4,9}$ Claudio Queiroz, \\ ${ }^{9}$ Jose Junior, ${ }^{3,4,7}$ Pedro Ribeiro and ${ }^{1,2}$ Antonio E. Nardi \\ ${ }^{1}$ Panic and Respiration Laboratory, Institute of Psychiatry, \\ Federal University of Rio de Janeiro (IPUB/UFRJ), Brazil \\ ${ }^{2}$ National Institute for Translational Medicine (INCT-TM), Brazil \\ ${ }^{3}$ Institute of Applied Neuroscience (INA), Rio de Janeiro, Brazil \\ ${ }^{4}$ Brain Mapping and Sensory Motor Integration, Institute of Psychiatry of \\ Federal University of Rio de Janeiro (IPUB/UFRJ), Brazil \\ ${ }^{5}$ Department of Neurology, Philipps University-Marburg, Marburg, Germany \\ ${ }^{6}$ Faculty of Psychology, Brazilian Institute of Medicine and \\ Rehabilitation (IBMR), Rio de Janeiro, Brazil \\ ${ }^{7}$ Department of Bioscience (EEFD/UFRJ), School of Physical Education, Brazil \\ ${ }^{8}$ Department of Psychology, Pontifical Catholic University of Rio de Janeiro, Brazil \\ ${ }^{9}$ Department of Physical Therapy, Veiga de Almeida University, Rio de Janeiro, Brazil
}

\begin{abstract}
Problem statement: Repetitive Transcranial Magnetic Stimulation (rTMS) is a noninvasive procedure whereby a pulsed magnetic field stimulates electrical activity in the brain. Dystonia is characterized by several disabling symptoms for which effective, mechanism-based treatments remain elusive. Approach: Consequently, more advanced non-invasive therapeutic methods were required. A possible method to modulate brain activity and potentially viable for use in clinical practice was rTMS. We focus on the basic foundation of rTMS, the effects of rTMS on neuroplasticity and sensorimotor integration and the experimental advances of rTMS that may become a viable clinical application to treat dystonia. Results: The findings showed that rTMS can improve some symptoms associated with dystonia and might be useful for promoting cortical plasticity in dystonic patients. These changes were transient and it is premature to propose these applications as realistic therapeutic options, even though the rTMS technique has shown itself to be, potentially, a modulator of sensorimotor integration and neuroplasticity. Conclusion/Recommendations: Functional imaging of the region of interest could highlight the capacity of rTMS to bring about plastic changes of the cortical circuitry and hint at future novel clinical interventions. We recommend further studies to clearly determine the role of rTMS in the treatment of these conditions. Finally, we must remember that however exciting the neurobiological mechanisms might be, the clinical usefulness of rTMS will be determined by their ability to provide patients with neurological and psychiatric disorders with safe, long-lasting and substantial improvements in quality of life.
\end{abstract}

Key words: Brain tissue, dystonia, neuroplasticity, Repetitive Transcranial Magnetic Stimulation (rTMS), sensorimotor integration, Transcranial Magnetic Stimulation (TMS)

\section{INTRODUCTION}

Dystonia is a movement disorder characterized by sustained contractions of agonist and antagonist muscles leading to abnormal twisting movements and postures. Although dystonia is generally regarded as a pure motor disorder, due to a dysfunction in the cortical-striatal-thalamic-cortical motor loop (Berardelli et al., 1998), it is commonly preceded by sensory symptoms, such as, discomfort, pain, or kinaesthetic

Corresponding Author: Sergio Machado Panic and Respiration Lab, Institute of Psychiatry, Federal University of Rio de Janeiro (IPUB/UFRJ), Brazil, Av. Venceslau Brás 71, Fundos. Botafogo, CEP 22290-140 Tel: +5521-78719183 Fax: +5521-25112254 
sensations (Ghika et al., 1993). More specifically, focal hand dystonia is a form of idiopathic adult onset dystonia and tends to be task specific involving repetitive fine movements of the hand such as playing an instrument, writing or typing (Hallett, 1998).

Electrophysiological findings of distonic patients, in general primary dystonia, have identified widespread abnormalities of inhibitory motor circuits in the brain, brainstem and spinal cord (Berardelli et al., 1998). Moreover, data from neuroimaging studies have also shown increases in blood flow or glucose metabolism in several brain regions, including the Prefrontal Cortex (PFC), cerebellum, Insular Cortex (IC), Parietal Cortex (PC) and Supplementary Motor Area (SMA) (Eidelberg et al., 1998).

Some of these abnormalities were only present during movement (Ceballos-Baumann et al., 1995; Machado et al., 2010). As a result, Transcranial Magnetic Stimulation (TMS) has been postulated as a potential candidate to reduce this abnormal cortical excitability and, potentially, have an effect on symptoms. TMS in its repetitive form, i.e., rTMS, can modulate cortical excitability beyond the period of stimulation itself (O'Readon et al., 2006). Depending on essential stimulation frequency parameters and on the number of trains of stimuli delivered, rTMS can produce lasting up- or down-regulation of the corticospinal system (Maeda et al., 2000). TMS is based on Faraday's law of electromagnetic induction by which electrical activity in the brain tissue can be influenced by the magnetic field, thereby inducing electrical current that depolarizes neurons (Tyc and Boyadjian, 2006). The application of rTMS generates clear effects on a range of measures of brain function and has become an important research tool due to its potential application as a clinical treatment for a variety of neurological and psychiatric disorders, for instance depression (Hoppner et al., 2010; SchonfeldtLecuona et al., 2010). Within this context, the use of rTMS is considered a brain-system-based neuromodulation treatment due to its focus on directly targeting the neural circuitry of the disorders. rTMS acts altering or modulating the function of the neural circuitry in the brain that is believed to be disorganized in certain disorders (Nahas et al., 2001; Speer et al., 2000).

Although this rationale was behind most of the rTMS studies in dystonia, developments in understanding the pathophysiology of dystonia have shown that dystonia could have its pathological basis in the enhanced ability of the brain to undergo plastic change (Edwards et al., 2006; Quartarone et al., 2006). From a clinical point of view, dystonia occurs after intense practice of complex movements in both human beings (Frucht, 2004) and animals (Byl et al., 1996). Dystonia is triggered or worsened by injury that increases long-term potentiation (LTP) in the cortex that corresponds to the injured limb. From an experimental point of view, dystonia is associated with an excessive response to several plasticity-inducing protocols, e.g., rTMS (Quartarone et al., 2005). These data support the hypothesis that in dystonia there is an increased tendency to form associations between inputs and outputs, which could lead to abnormal unwanted connections and subsequent impairment of motor control. Dystonia is, therefore, a candidate for the therapeutic use of rTMS.

In fact, there is now a growing interest in the research of new treatment for neurological and psychiatric disorders, however, the main focus of the possible therapeutic effects of rTMS is still in the domain of depression (Hoppner et al., 2010, Schonfeldt-Lecuona et al., 2010). Within this context, this review paper aims to provide information on the current research and main findings related to the potential therapeutic effects of rTMS on dystonia.

Foundations of repetitive transcranial magnetic stimulation (rTMS): rTMS is the application to a certain brain area of a train of repeated TMS pulses with the same intensity at a given frequency (Machado et al., 2008). TMS was originally introduced by Anthony Barker et al. (1985) as noninvasive focal brain stimulation, safe and painless way to study the CNS. Transcranial magnetic stimulation exploits the principle of inductance discovered by Michael Faraday in 1838 (i.e., Faraday's law of electromagnetic induction) where an electrical current is applied over the scalp and skull in order to transmit electrical energy through a magnetic coil (Cheng and Chan, 2010).

The TMS device essentially comprises a capacitor and stimulating coil connected to the stimulator, usually round or figure-eight (butterfly) in shape. The coil is placed on a subject's head and as a brief pulse of current flows through it, a rapid time-varying magnetic field is generated which passes through the subject's skull and induces a current in the conductive brain tissue (Fig. 1). 


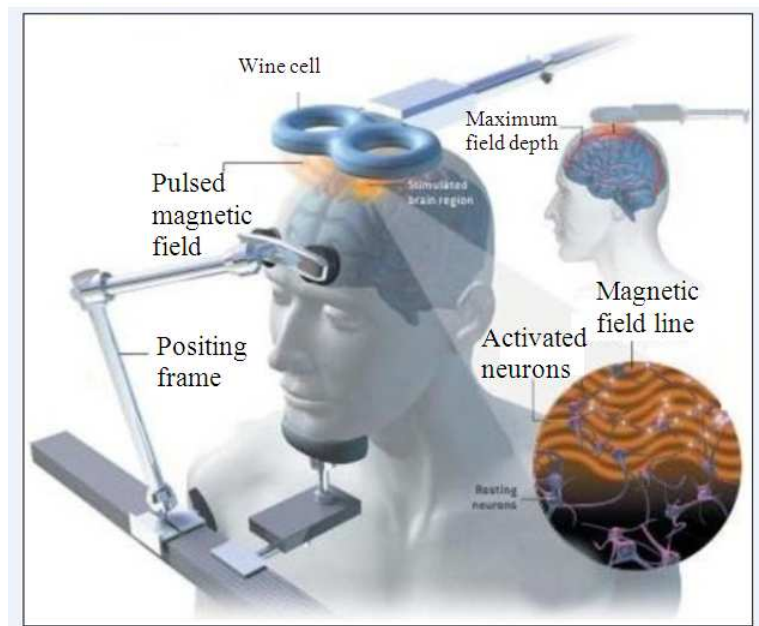

Fig. 1: Repetitive transcranial magnetic stimulation (rTMS) in humans. According to the evidence cited in this review, there are basically two types of coils: round coils which are relatively non focal and figure eight-shaped coils used to stimulate specific areas, producing maximal current at the intersection of the two round components. The modulatory effects of rTMS depend particularly on the intensity, frequency, train length, intertrain-interval, total number of magnetic pulses delivered in the stimulation session, as well as on the coil configuration, current direction, pulse waveform and position of the coil with respect to the cortex. In humans, the area of stimulation depends on the shape of the coil and the stimulation intensity

The figure-eight coil produces a focal field allowing fairly detailed mapping of cortical representation (Hallett, 2007; Kobayashi and PascualLeone, 2003; Pascual-Leone and Tormos-Munoz, 2008). The maximal field strength generated by commercially available stimulators is in the 2 Tesla (T) range and they are able to activate cortical neurons at a depth of $1.5-2 \mathrm{~cm}$ beneath the scalp. The precise effect of the stimulation on neuronal activity remains unclear. It is supposed that the magnetic stimulus (duration of $100 \mu$ s) synchronously excites a population of neurons, inducing rapid changes in the firing rates of certain neural networks during only a few milliseconds (Maeda et al., 2000).

rTMS can be classified as "high-frequency rTMS" $(>1 \mathrm{~Hz})$ or "low-frequency rTMS" $(\leq 1 \mathrm{~Hz})$. Although the response to rTMS can vary across individuals (Hallett, 2000), high-frequency rTMS seems to facilitate cortical excitability, while low-frequency
rTMS can suppress this excitability on the motor cortex (Hallett, 2007, Barker et al., 1985). Recently, a novel pattern of rTMS called Theta-Burst Stimulation (TBS) was developed to produce changes in the human cerebral cortex excitability (Kobayashi and PascualLeone, 2003). The main advantage of TBS paradigm as compared with conventional rTMS protocols is that a shorter period (between 20 and 190 s) of subthreshold stimulation causes changes in cortical excitability that outlast the time of stimulation for at least 15-20 min. Huang et al. (2005) proposed a TBS protocol consisting of bursts of 3 pulses given at $50 \mathrm{~Hz}$ repeated every 200 $\mathrm{ms}(5 \mathrm{~Hz})$, thus, mimicking the coupling of theta and gamma rhythms in the brain. Two main modalities of TBS have been tested. Intermittent TBS (iTBS) induces facilitation of motor cortical excitability whereas continuous TBS (cTBS) leads to inhibition for 15-30 min after application (Huang et al., 2005). Motor cortical excitability is characterized in surface electromyographic recordings considering Motor Evoked Potentials (MEPs) amplitude. The most common value is the resting motor threshold (rMT) measured with relaxed muscles. It is defined as the minimum amount of energy (i.e., intensity of stimulation) needed to induce a MEP in a hand muscle in at least 5 out of 10 consecutive trials (Pascual-Leone et al., 2000; Pascual-Leone and Tormos-Munoz; 2008; Rossini et al., 1994; 2010; Walsh and Rushworth, 1999). RMT is additionally used to establish the individual intensity of stimulation, usually described as a percentage of the device's available output (Rossini et al., 1994; 2010).

However, the mechanisms underlying rTMS protocols remain still unclear. It has been suggested that LTP- and long-term depression (LTD)-like mechanisms as well as GABAergic activity are involved in the effects of rTMS protocols (Ziemann, 2004; Thickbroom, 2007). Animal studies suggest that modulation of neurotransmitters and gene induction may contribute to the long-lasting modulatory effects of rTMS (Hallett, 2007; Kobayashi and Pascual-Leone, 2003; Arias-Carrion, 2008; Aydin-Abidin et al., 2008; Murillo-Rodriguez et al., 2009).

\section{Neuroplasticity induced by repetitive Transcranial} Magnetic Stimulation (rTMS): Transcranial magnetic stimulation can be used in a variety of ways to induce plastic changes in the brain and can thus be exploited to assess the brain's capacity for plasticity. Additionally, induced plastic changes can be exploited therapeutically and this aspect will be discussed below. Although rTMS is sometimes used to disrupt cortical activity for long periods, the majority of applications take 
Am. J. Neuroscience 2 (1): 5-16, 2011

advantage of the fact that longer periods of rTMS can sometimes produce effects on cortical circuits that outlast the duration of the stimulus (Hallett et al., 1999). This, in fact, makes it possible to provoke and study mechanisms of acute cortical reorganization in the healthy human brain. Most descriptive studies of the effects of rTMS have used the primary motor cortex and have shown that rTMS can have long-term effects on corticospinal excitability, but also that the direction, magnitude and duration of the conditioning effects are critically dependent on the stimulation variables.

Three factors influence the effect of rTMS: frequency, intensity and duration of the stimulation. It is thus important to specify all three of these parameters when describing the results of any rTMS experiment. An effective way of modulating synaptic efficacy is to activate a cell with two or more inputs, almost simultaneously. If the impulses are transmitted along the same synaptic pathway, the stimulation is referred to as homosynaptic, conversely, if they travel along different synaptic pathways, it is termed heterosynaptic (Hallett, 2007). In general, when authors talk of "highfrequency stimulation", they are referring to frequencies of about $5 \mathrm{~Hz}$ and above, "low-frequency stimulation" instead refers to frequencies of about $1 \mathrm{~Hz}$.

As regards the strength of stimulation, rTMS at an intensity of more than about $10 \%$ above the MEP threshold in relaxed muscle is classed as "high-intensity stimulation" or suprathreshold rTMS. High frequencies of rTMS, especially at suprathreshold, produce facilitatory aftereffects on corticospinal excitability (Maeda et al., 2000). A 10-pulse rTMS train at $150 \%$ resting motor threshold and $20 \mathrm{~Hz}$ caused an increase in MEP size lasting about three minutes after the administration of rTMS (Pascual-leone et al., 1994). A 30-pulse rTMS train at $120 \%$ resting motor threshold and $15 \mathrm{~Hz}$ caused a shorter and smaller increase in MEP size lasting $90 \mathrm{sec}$ (Wu et al., 2000).

In the case of stimulation at intensities below the resting motor threshold, longer trains are usually required before any lasting effect is seen. For example, Maeda et al. (2000) reported a facilitation of MEPs lasting two minutes after the administration of 240 pulses of $20 \mathrm{~Hz}$ stimuli at $90 \%$ resting threshold. Notably $10 \mathrm{~Hz}$ rTMS had no lasting effect on MEP size. Low-frequency rTMS usually results in suppression of corticospinal excitability (Hallett, 2007). A 15-min train of $0.9 \mathrm{~Hz}$ applied at $115 \%$ motor resting threshold over the primary motor cortex (M1) reduced corticospinal excitability (i.e., it increased the resting motor threshold and suppressed the MEP input-output curve) for at least 15 minutes after the end of stimulation (Muellbacher $e t$ al., 2002).
Low-frequency rTMS at intensities below the rMT have a much weaker effect on corticospinal excitability as compared with suprathreshold rTMS (Fitzgerald et al., 2002). Even lower intensities (90\% active motor threshold-aMT) or lower frequencies $(0.1 \mathrm{~Hz})$ had no lasting effect (Gerschlager et al., 2001). The duration of rTMS affects the duration and depth of the after-effect. Maeda et al. (2000) and Touge et al. (2001) both used $1 \mathrm{~Hz}$ rTMS, at $90 \%$ and $95 \%$ resting threshold respectively. Longer periods of rTMS lead to longer and stronger reductions in excitability. Studies both of relatively short trains $(<20$ stimuli) and of longer trains of rTMS provide an insight into the interaction between factors promoting inhibition and factors promoting excitation.

If the number of stimuli in the train was increased to 20, facilitation became prominent at high intensities (Modugno et al., 2001). It was suggested that the threshold for inhibitory effects was lower than that for facilitatory effects and that inhibition built up faster than facilitation. The result of this was that short trains tended to result in transient inhibition, whereas longer trains were likely to produce facilitation, particularly if the intensity and frequency of stimulation were high.

The potentially restorative effects of rTMS have also been tested in patients with motor cortex damage, investigating whether function can be restored and plasticity induced in patients with neglect. A recent study investigated repetitive stimulation of the contralesional hemisphere as a means of restoring interhemispheric inhibitory balances and consequently motor function and behavior (Mansur et al., 2005) and showed that repetitive stimulation of the contralesional motor cortex with low frequencies led to subsequently improved motor functions. It is important to note that this rTMS-induced improvement occurred only when stimulating over the contralesional motor cortex and not with premotor cortex or sham stimulation.

Research to establish the optimal parameters for the most effective and efficient induction of neuroplasticity remains to be completed. It is known that higher-frequency rTMS over the more injured M1 can, compared to sham rTMS, lead to improved motor function. Thus, motor plasticity and improved outcome with rTMS can be induced either by low-frequency rTMS over the less injured hemisphere or highfrequency rTMS over the more injured hemisphere. Takeuchi et al. (2005) and Fregni et al. (2006) evaluated the effects of low-frequency rTMS of the intact hemisphere after this suppressive protocol of motor cortex excitability. Takeuchi et al. (2005) observed a reduction of the transcallosal inhibition from the intact hemisphere in response to rTMS of the 
damaged M1 and Fregni et al. (2006) a reduction of the MT in response to rTMS of the damaged motor cortex. Kim et al. (2006) showed that high-frequency rTMS of the damaged M1 increased MEP amplitude. Talelli et al. (2007) evaluated the effects of a single session of rTMS, using TBS. They found that MEP amplitude was increased on the stroke side after TBS of the stroke hemisphere. The effects produced by rTMS on M1 excitability in patients with acute stroke are still unknown. Taken together, these findings using rTMS highlight the vast potential offered by this relatively new technology for assessing and promoting neuroplasticity and rehabilitation.

\section{Sensorimotor integration process and repetitive Transcranial Magnetic Stimulation (rTMS):} Sensorimotor integration is the continuous processing, by the motor system, of sensory afferents in order to prepare motor acts and to enhance the execution of fine motor activities. In this process, the Central Nervous System (CNS) integrates information coming from multiple sensory channels, allowing the performance of specific, goal-directed tasks (Machado et al., 2010). This process has been documented in the intact human cortex through experiments using TMS. The cerebral cortex is composed of cortical areas that are neither purely sensory nor purely motor, but associative and serve higher-order integrative functions. These higherorder areas of the cortex called association areas, associate sensory inputs with motor response and perform those mental processes that intervene between sensory inputs and motor outputs (Miller and Cohen, 2001).

Accordingly, there have been reports that alterations of sensory input may influence the excitability of projections to muscles in the opposite arm. Werhahn et al. (2002) found that anesthesia of the hand and forearm of one hand increased MEPs of hand muscles in the opposite hand and pharmacological studies suggested that this effect might be GABAdependent. In addition, the authors found that excitability of the M1 in the hemisphere contralateral to the anesthetized limb was reduced compared with excitability of the M1 in the 'intact' hemisphere. Kossev et al. (2001) showed that enhancing, rather than decreasing, sensory input can have effects on the excitability of corticospinal projections to the opposite arm. It thus emerges that manipulations of sensory inputs can be used to induce lasting changes in motor cortical outputs. Reduction of afferent input by anesthesia causes disinhibition within the M1 (Werhahn et al., 2002) which can be associated with improved hand function after stroke (Floel et al., 2004). Increased sensory input can likewise be used to increase motor cortical output (Conforto et al., 2002). Muscle vibration itself can induce changes in associations between cortical hand muscle representations (Rosenkranz et al., 2004). Sensorimotor integration is known to function abnormally in types of dystonia (Rosenkranz et al., 2005), while stroke is associated with defects in short intracortical inhibition (SICI) and interhemispheric inhibition (Butefisch et al., 2008). The inhibitory imbalance between the unaffected and affected hemispheres following stroke is a reasonable target for therapeutic modulation. The fact that muscle vibration has effects not only in the contralateral but also the ipsilateral hemisphere and moreover can modulate the relationship between the two, expands the scope for targeted interventions designed to redress inhibitory imbalances in these disorders.

Relevant methodological aspects: sham-rTMS and stimulation parameters: An important issue in the TMS research regarding the design of randomized, sham-controlled clinical trials is the use of appropriate control conditions that provide a reliable blinding of patients and investigators (De Graaf and Sack, 2011). Within this context, different control conditions can be used to try and ensure that changes in performance be ascribed to rTMS effects upon a specific brain area. One of the most common strategies is the use of sham stimulation (sham-rTMS) (Sandrini et al., 2011). rTMS is indeed associated with a number of sensory perceptions that can nonspecifically interfere with task performance. For instance, the discharging coil produces a click sound that may induce arousal, thereby modulating task performance, irrespective of the experimental demands (i.e., via intersensory facilitation) (Marzi et al., 1998). An alternative way that is routinely used in the cognitive TMS literature is vertex stimulation because the auditory and somatosensory activations caused by vertex TMS can be equivalent to those of real TMS. Of course, the underlying assumption is that vertex TMS does not affect the cognitive network active during task execution (Dormal et al., 2008; Knops et al., 2006).

In general, sham-rTMS has been applied by tilting the coil away from the scalp (Sandrini et al., 2004), so that both sound and scalp contact are roughly similar to those experienced during active stimulation, whereas the magnetic field does not reach cortical neurons or cutaneous receptors or superficial muscles. Although sham coils produce an analogous sound artifact, they do not induce the same scalp sensations or muscle twitches, so that they can rest tangential to the scalp surface, exactly as they are during active stimulation 
Am. J. Neuroscience 2 (1): 5-16, 2011

(Cappelletti et al., 2007; Cohen et al., 2007). Another important consideration that must be taking into account in order to determine the specific efficacy of rTMS in clinical trials and to create a credible placebo (i.e., sham-rTMS) condition is that patients in randomized trials should be naive to rTMS, in other words, rTMS studies should not have a crossover design. With respect to this issue, the ideal sham condition should not have a real stimulation effect and it should not be recognized as sham by patients, particularly when considering that real stimulation conditions come along with rTMS specific side effects.

In line with that, Herwig et al. (2010) investigating the antidepressant effects of rTMS, asked for patients to give their impression whether they received the sham or the real treatment and if they would recommend the treatment to others. From 15 patients with real stimulation, 11 suggested that they obtained true stimulation and 4 to have obtained sham. From 14 sham stimulated subjects, 9 suggested that they obtained the real condition and 5 to have been sham stimulated. There was no significant difference between these and in addition, the majority of patients in both stimulation conditions would recommend rTMS to others. In both conditions, the majority of subjects believed they had received the real condition. This implies suitability of the sham condition used since subjects appeared not to be able to accurately identify or differentiate this condition from sham. The results imply the feasibility of a valid sham condition with a "real" coil.

However, there is evidence that some types of sham manipulations used in clinical trials actually do exert some effects on the brain (Lisanby et al., 2001; Loo et al., 2000). The tilting does reduce any discomfort from scalp stimulation associated with active rTMS and, thus, may have the potential to interfere to some degree with the adequacy of study blinding. Studies guard against this by recruiting only rTMS-naïve patients, so that subjects are not cued to discriminate between active and sham conditions based on scalp sensation. Even if a form of coil-tilt sham that does not exert measurable brain effects is used, studies rarely report data on the integrity of the blind on the part of the patients and raters. It is reasonable to assume that crossover trials with coil-tilt sham conditions are likely to be unblinded because active and sham rTMS do not feel the same (Tsubokawa et al., 1993; Shah et al., 2008). Other option include the one used in a recent experiment consisting of a sensor strip between the electromagnet and the scalp, which can counterstimulate during pulse delivery so as to reduce the scalp sensation perceived from active rTMS (O'Reardon et al., 2007).
The matter of placebo effects is especially important in some conditions, such as studies investigating the efficacy of treatments (Sandrini et al., 2011). For such purposes alternative methods of brain stimulation to provide suitable control conditions have been proposed. For instance, Rossi et al. (2007) developed a new method of sham stimulation, known as real electromagnetic placebo, in which a fake coil (made of wood) with the same shape as a real coil is attached to the real coil. This fake coil has two functions: to block the magnetic field from the real coil and to house a bipolar electrical stimulator in contact with the scalp. This device is more likely to be judged as real stimulation by naive TMS subjects. The difficulty in blinding TMS makes the comparison of TMS with a gold standard treatment (e.g., psychopharmacology) complex. In the case of pharmacologic agents, it would be possible to use a "double-dummy" design in which some patients would receive sham rTMS plus active medication, whereas other patients would receive active rTMS and a placebo pill. An additional challenge in the design of clinical trials with rTMS pertains to the standardization of the dosage. Just as it is critical to control the dosage of medication administered during drug trials, it is likewise essential to control the amount of rTMS administered and the location of the brain region stimulated (Lisanby et al., 2002).

Other important considerations to be taken into account are the parameters of stimulation, e.g., pulse width, number of stimulation sessions, frequency, intensity and site of stimulation (Dileone et al., 2010). A protocol composed of repeated sessions may be superior to a single session, due to its cumulative effect related to amount of stimulation required to induce a sustained effect. Indeed, although some studies have shown a relatively long-lasting effect (i.e., of 2 weeks), this period is short if the goal is to induce a clinically meaningful result. Maintenance treatments or other patterns of stimulation that might induce longer-lasting modulation of cortical excitability should be explored. One possibility is to increase the total number of sessions, as in a recent study of major depression, in which up to 30 sessions of rTMS were administered (Fitzgerald et al., 2006). Novel patterns of stimulation, for example primed $1 \mathrm{~Hz}$ stimulation (Iyer et al., 2003) or theta burst stimulation (Huang et al., 2005), might offer advantages, as they seem to induce longer-lasting long-term-depression-like phenomena. Careful consideration of cortical targets seems to be critical and this might need to be individualized for each patient and underlying pathology. 
In summary, a number of parameters need to be taken into account in order to optimize the clinical effects of rTMS. Predictions with regard to the efficacy of clinical effects of rTMS are hampered due to the relative paucity of parametric studies performed on these variables. Moreover, individualizing stimulation parameters, taking into account the underlying pathophysiology and the stimulation settings by online physiological and neuroimaging measures, seems to be a crucial procedure to adopt (De Graaf and Sack, 2011; Sandrini et al., 2011).

Repetitive Transcranial Magnetic Stimulation (rTMS) for the treatment of Dystonia: Long-lasting influences on the brain depend on changes in synaptic strength or anatomical changes (e.g., alterations in dendritic spines or sprouting) and since such anatomical changes may be a secondary consequence of extended changes in synaptic strength, the aim of rTMS is to alter synaptic strength. This effect has been seen both in neurological and psychiatric disorders. This modulation of cortical activity induced by rTMS is not limited only to motor areas. There is also evidence that its longlasting effects can be provoked in areas outside the motor cortex and be associated with assessable behavioral changes (Hallett, 2007; Kobayashi and Pascual-Leone, 2003). This finding raises the possibility of therapeutic applications of rTMS in order to "normalize" pathologically decreased or increased levels of cortical activity. Therefore, in this section, we will discuss the use of rTMS as a potential treatment of neurological and psychiatric disorders.

The sensorimotor integration is a process that through complex neural operations in the brain, allows the execution of a certain voluntary motor behavior in response to specific demands of the environment. In other words, it is the dynamic combination of sensory input into intentional motor response, intending to prepare motor acts to improve the performance of certain motor tasks (Machado et al., 2010; Abbruzzese and Berardelli, 2003). Therefore, the motor behavior of healthy subjects or patients with movement disorders (e.g., dystonia) depends on the sensorimotor integration process.

Many studies deal with the sensorimotor integration process to reveal the brain functions related to the pathophysiological mechanisms of dystonia and to improve neurofunctional rehabilitation strategies based on the capacity to rearrange the CNS. The appropriate execution of a voluntary movement depends considerably also on peripheral sensory feedback. Thus, peripheral pathways transmit sensory information to M1. Abnormalities in the peripheral afferent input or in the brain response to sensory input may interrupt the neural networks processing in the sensoriomotor areas of the cerebral cortex. Augmented evidence of the involvement of the sensory system in pathophysiology dystonia is crucial to consider the possible contribution of changes in sensorimotor integration, i.e., the capacity to exploit sensory information accurately for assisting neural networks responsible for an appropriate movement execution (Machado et al., 2010; Abbruzzese and Berardelli, 2003).

Within this context, the use of rTMS in treatment of sensorimotor deficits of dystonia as therapeutic tool would require repetitive and frequent use to be effective. rTMS has the potential to fulfill adjunctive treatment role in rehabilitation of sensorimotor deficits in dystonia by a rational and selective modulation of symptoms and their underlying neuropathophysiology on an individual basis (Machado et al., 2008). Hypothetically, rTMS must be applied over selected cortical regions in order to modulating the specific cortical-subcortical networks possibly responsible for a given subset of symptoms. In this sense, rTMS could modulate cortical excitability, underlying adaptive and maladaptive plasticity (Williams et al., 2009).

There is a different rationale for the use of rTMS in dystonia in which physiological findings reveal a decrease in ICI. Since rTMS delivered over M1 at $1 \mathrm{~Hz}$ can induce an increase in inhibition, this effect might improve the deficit. An initial study showed a normalization of ICI and some modest improvement in performance (Siebner et al., 1999). This improvement of deficient ICI and of handwriting persisted, at most, for $3 \mathrm{~h}$ after application of a 30-min. train of rTMS but resulted in clinical benefits in only 2 of 16 patients studied (Kujirai et al., 1993). Although these effects are transient, the data support the concept of impaired inhibitory mechanisms in the M1. Another target could be the premotor cortex (PMC), since rTMS at $1 \mathrm{~Hz}$ can improve the deficit in reciprocal inhibition seen in dystonia (Huang et al., 2004). Accordingly, nine patients with writer's cramp and seven age-matched control subjects were studied using subthreshold $0.2 \mathrm{~Hz}$ rTMS applied to the M1, SMA, or PMC (Murase et al., 2005). Stimulation of the PMC but not of the M1 significantly improved the handwriting rating in the patient group. rTMS over the other sites or using a sham coil in the patient group and trials in the control group revealed no clinical changes.

In a recent experiment, Baumer et al. (2007) investigated whether, as hypothesized, functional alterations make the somatosensory cortex (S1) of writer's cramp patients more vulnerable to the 
inhibitory effects when a subthreshold $1 \mathrm{~Hz}$ rTMS is applied. Seven patients and eight healthy subjects were assessed. In addition, patients also were submitted to rTMS of M1. Short-latency afferent inhibition (SAI) was investigated in the relaxed first dorsal interosseous muscle through conditioning electrical stimulation of the index finger and rTMS pulses over the contralateral M1. Baseline SAI was not significantly different between groups, however, S1 but not M1 rTMS reduced SAI in the patients. Moreover, in the healthy subjects, rTMS had no effects on SAI, which is mediated mainly at the sensorimotor cortex. It was concluded that there was an irregular responsiveness of $\mathrm{S} 1$ to $1 \mathrm{~Hz}$ rTMS in the patients, which may be a trait suggestive of maladaptive plasticity in the sensorimotor areas in these subjects.

Gilio et al. (2007), on the other hand, verified whether $5 \mathrm{~Hz}$ rTMS obtains similar MEP facilitation during stimulation and similar facilitatory after-effects in patients with upper limb dystonia and in healthy subjects. Protocols of 5, 10 and 20 stimuli trains were distributed at $120 \%$ resting motor threshold over the M1 with the individuals at rest. The rTMS trains were followed by single test stimuli distributed at a variety of interstimulus intervals $(0.5-10 \mathrm{~s})$ at $120 \%$ rMT using a conditioning test paradigm. The effects of suprathreshold $1 \mathrm{~Hz}$ rTMS were also evaluated. The MEP amplitude during the course of the trains and of the test stimuli was measured. In control studies, the authors investigated the effect on the MEP amplitude of afferent inputs elicited by muscle twitches after ulnar nerve stimulation. Equally, the patients and the healthy participants showed significantly increased MEP amplitude over the course of the $5 \mathrm{~Hz}$ rTMS protocol. In addition, in both groups the MEP facilitation was found to outlast the $5 \mathrm{~Hz}$ rTMS, nevertheless the facilitatory after-effects were more evident and long-lasting in the patients. Moreover, it was also verified that MEP amplitudes during and after $1 \mathrm{~Hz}$ rTMS remained unchanged. Ulnar nerve stimulation did not change the test MEP amplitude. The authors concluded that patients with upper limb dystonia show an atypical recovery when assessed through MEP facilitation after suprathreshold $5 \mathrm{~Hz}$ rTMS application, indicating an atypical pattern of short-term cortical plasticity.

More recently, in order to clarifying the rationale for using rTMS in dystonia over dorsal premotor cortex (PMd), Huang et al. (2010) investigated how the motor system would react to cTBS with 300 and 600 pulses (cTBS300 and cTBS600) and its after-effects were quantified by measuring the amplitude of MEPs, Short Interval Intracortical Inhibition/Facilitation (SICI/ICF) within M1, Reciprocal Inhibition (RI) and writing tests comparing healthy individuals to patients with focal hand dystonia. The researchers found that cTBS300 and cTBS600 over PMd suppressed MEPs for $30 \mathrm{~min}$ or more and cTBS600 decreased SICI and RI. On the other hand, neither form of cTBS over PMd had any significant effect on MEPs, while cTBS600 increased effectiveness of SICI and RI and improved writing in distonic patients, suggesting that the reduced PMd to M1 interaction in dystonic patients is likely to be due to reduced excitability of PMd-M1 connections.

In another study, Huang (2010) discussed the importance of TBS and its capacity to produce plasticity-like effects more efficiently and powerfully than rTMS traditional protocols. The excitability of circuits within the M1 can be modified not only by TBS over but also when it is delivered to the PMC. Experiments using TBS over the M1 and PMC provide a better understanding of dystonia and the results also distinguish the different mechanisms of the effects of TBS given to the M1 and PMC. Therefore, these findings support the hypothesis that TBS is a potential therapeutic strategy to restore damaged motor functions.

\section{CONCLUSION}

Since its introduction 26 years ago, TMS has evolved into a sophisticated tool for neuroscience research. It is an excellent technique and complements other non-invasive methods for studying human brain physiology. The rTMS technique is a non-invasive and effective methodology with potential for therapeutic use. In this review, we have focused in dystonia, which have showed rTMS can improve some symptoms associated with these conditions. rTMS may become an additional tool for early neurorehabilitation and might be useful for promoting cortical plasticity in dystonic patients. However, these changes are transient and it is premature to propose these applications as realistic therapeutic options, even though the rTMS technique has shown itself to be, potentially, a modulator of sensorimotor integration and neuroplasticity. Functional imaging of the region of interest could highlight the capacity of rTMS to bring about plastic changes of the cortical circuitry and hint at future novel clinical interventions. As new coils and new patterns of stimulation are developed, we are likely to see the emergence of even more innovative ways of using this technique. Combined non-invasive techniques can be used in imaginative ways. In this manner, electroencephalography could be used to establish exactly where and when to deliver a TMS pulse in order to obtain maximum advantage. Although further 
developments are needed to make the effects more robust and longer lasting, future work in this area promises to advance our understanding of the pathophysiology of dystonia, generate widely applicable diagnostic tools for clinical neurophysiology and perhaps establish neuromodulation as a viable therapeutic option in neurorehabilitation. Thus, we recommend further studies to clearly determine the role of rTMS in the treatment of these conditions. Finally, we must remember that however exciting the neurobiological mechanisms might be, the clinical usefulness of rTMS will be determined by their ability to provide dystonic patients with safe, long-lasting and substantial improvements in quality of life.

\section{REFERENCES}

Abbruzzese, G. and A. Berardelli, 2003. Sensorimotor integration in movement disorders. Mov. Disord., 18: 231-240. PMID: 12621626

Arias-Carrion, O, 2008. Basic mechanisms of rTMS: Implications in Parkinson's disease. Int. Arch. Med., 1: 1-2. PMID: 18471317

Aydin-Abidin, S., J. Trippe, K. Funke, U.T. Eysel and A. Benali, 2008. High-and low-frequency repetitive transcranial magnetic stimulation differentially activates c-Fos and zif268 protein expression in the rat brain. Exp. Brain Res., 188: 249-261. PMID: 18385988

Barker, A.T., R.. Jalinous and I.L. Freeston, 1985. Noninvasive magnetic stimulation of human motor cortex. Lancet, 1: 1106-1107. PMID: 2860322

Baumer, T., C. Demiralay, U. Hidding, R. Bikmullina and R. Helmich et al., 2007. Abnormal plasticity of the sensorimotor cortex to slow repetitive transcranial magnetic stimulation in patients with writer's cramp. Mov. Disord., 22: 81-90. PMID: 17089385

Berardelli, A., J.C. Rothwell, P.D. Thompson, M. Manfredi and C.D. Marsden, 1998. The pathophysiology of primary dystonia. Brain, 121: 1195-1212. PMID: 9679773

Butefisch, C.M., M. Wessling, J. Netz, R.J. Seitz and V. Homberg, 2008. Relationship between interhemispheric inhibition and motor cortex excitability in subacute stroke patients. Neurorehabil. Neural Repair, 22: 4-21. PMID: 17507644

Byl, N.N., M.M. Merzenich and W.M. Jenkins, 1996. A primate genesis model of focal dystonia and repetitive strain injury: I. Learning-induced dediff erentiation of the representation of the hand in the primary somatosensory cortex in adult monkeys. Neurology, 47: 508-520. PMID: 8757029

Cappelletti, M., H. Barth, F. Fregni, E.S. Spelke and A. Pascual-Leone, 2007. rTMS over the intraparietal sulcus disrupts numerosity processing. Exp. Brain Res., 179: 631-642. PMID: 17216413

Ceballos-Baumann, A.O., R.E. Passingham, T. Warner, E.D. Playford and Marsden et al., 1995. Overactive prefrontal and underactive motor cortical areas in idiopathic dystonia. Ann. Neurol., 37: 363-372. PMID: 7695236

Cheng, K. and C.H. Zou, 2010. BioInfoPhysics models of neuronal signal processes based on theories of electromagnetic fields. Am. J. Neurosci., 1: 13-20. DOI: 10.3844/ajnsp.2010.13.20.

Cohen, K.R., K.K. Cohen, T. Schuhmann, A. Kaas and R. Goebel et al., 2007. Virtual dyscalculia induced by parietal-lobe TMS impairs automatic magnitude processing. Curr. Biol., 17: 689-693. PMID: 17379521

Conforto, A.B., A. Kaelin-Lang and L.G. Cohen, 2002. Increase in hand muscle strength of stroke patients after somatosensory stimulation. Ann. Neurol., 51: 122-125. PMID: 11782992

De Graaf, T.A. and A.T. Sack, 2011. Null results in TMS: From absence of evidence to evidence of absence. Neurosci. Biobehav. Rev., 35: 871-877. PMID: 20955732

Dileone, M., P. Profice, F. Pilato, F. Ranieri and F. Capone et al., 2010. Repetitive transcranial magnetic stimulation for ALS. CNS Neurol. Disord. Drug Targets, 9: 331-334. PMID: 20406177

Dormal, V., M. Andres and M. Pesenti, 2008. Dissociation of numerosity and duration processing in the left intraparietal sulcus: A transcranial magnetic stimulation study. Cortex, 44: 462-469. PMID: 18387579

Edwards, M.J., Y.Z. Huang, P. Mir, J.C. Rothwell and K.P. Bhatia, 2006. Abnormalities in motor cortical plasticity differentiate manifesting and nonmanifesting DYT1 carriers. Mov. Disord., 21: 2181-2186. PMID: 17078060

Eidelberg, D., J.R. Moeller, A. Antonini, K. Kazumata and T. Nakamura et al., 1998. Functional brain networks in DYT1 dystonia. Ann. Neurol., 44: 303-312. PMID: 9749595

Fitzgerald, P.B., J. Benitez, A. de Castella, Z.J. Daskalakis and T.L. Brown et al., 2006. A randomized, controlled trial of sequential bilateral repetitive transcranial magnetic stimulation for treatment-resistant depression. Am. J. Psychiatry, 163: 88-94. PMID: 16390894 
Fitzgerald, P.B., T.L. Brown, Z.J. Daskalakis, R. Chen and Kulkarni, J., 2002. Intensity-dependent effects of $1 \mathrm{~Hz}$ rTMS on human corticospinal excitability. Clin. Neurophysiol., 113: 1136-1141. PMID: 12088710

Floel, A., U. Nagorsen, K.J. Werhahn, S. Ravindran and N. Birbaumeret et al., 2004 Influence of somatosensory input on motor function in patients with chronic stroke. Ann. Neurol., 56: 206-212. PMID: 15293272

Fregni, F., P.S. Boggio, A.C. Valle, R.R. Rocha and J. Duarte et al., 2006. A sham-controlled trial of a 5day course of repetitive transcranial magnetic stimulation of the unaffected hemisphere in stroke patients. Stroke, 37: 2115-2122. PMID: 16809569

Frucht, S.J., 2004. Focal task-specific dystonia in musicians. Adv. Neurol., 94: 225-230. PMID: 14509677

Gerschlager, W., H.R. Siebner and J.C. Rothwell, 2001. Decreased corticospinal excitability after subthreshold $1 \mathrm{~Hz}$ rTMS over lateral premotor cortex. Neurology, 57: 449-455. PMID: 11502912

Ghika, J., F. Regli and J.H. Growdon, 1993. Sensory symptoms in cranial dystonia: a potential role in the etiology? J. Neurol. Sci., 116: 142-147. PMID: 8336160

Gilio, F., A. Suppa, M. Bologna, C. Lorenzano and G. Fabbrini et al., 2007. Short-term cortical plasticity in patients with dystonia: A study with repetitive transcranial magnetic stimulation. Mov. Disord., 22: 1436-1443. PMID: 17516450

Hallett, M, E.M. Wassermann, A. Pascual-Leone and J. Valls-Sole, 1999. Repetitive transcranial magnetic stimulation. The International Federation of Clinical Neurophysiology. Electroencephalogr. Clin. Neurophysiol. Suppl., 52: 105-113. PMID: 10590981

Hallett, M., 1998. Physiology of dystonia. Adv. Neurol., 78: 11-18. PMID: 9750898

Hallett, M., 2000. Transcranial magnetic stimulation and the human brain. Nature, 406: 147-150. PMID: 10910346

Hallett, M., 2007. Transcranial magnetic stimulation: a primer. Neuron, 55: 187-199. PMID: 17640522

Herwig, U., L. Cardenas-Morales, B.J. Connemann, T. Kammer and C. Schonfeldt-Lecuona, 2010. Sham or real-post hoc estimation of stimulation condition in a randomized transcranial magnetic stimulation trial. Neurosci. Lett., 471: 30-33. PMID: 20064587

Hoppner, J., C. Berger, U. Walter, F. Padberg and J. Buchmann et al., 2010. Influence of repetitive transcranial magnetic stimulation on special symptoms in depressed patients. Restor. Neurol. Neurosci., 28: 577-586. PMID: 20714080
Huang, Y.Z., 2010. The modulation of cortical motor circuits and spinal reflexes using theta burst stimulation in healthy and dystonic subjects. Restor. Neurol. Neurosci., 28: 449-457. PMID: 20714069

Huang, Y.Z., J.C. Rothwell, C.S. Lu, J. Wang and R.S. Chen, 2010. Restoration of motor inhibition through an abnormal premotor-motor connection in dystonia. Mov. Disord., 25: 696-703. PMID: 20309999

Huang, Y.Z., M.J. Edwards, E. Rounis, K.P. Bhatia and J.C. Rothwell, 2005. Theta burst stimulation of the human motor cortex. Neuron, 45: 201-206. PMID: 15664172

Huang, Y.Z., M.J. Edwards, K.P. Bhatia and J.C. Rothwell, 2004. One-Hz repetitive transcranial magnetic stimulation of the premotor cortex alters reciprocal inhibition in DYT1 dystonia. Mov. Disord., 19: 54-59. DOI: 10.1002/mds.10627

Iyer, M.B., N. Schelper and E.M. Wassermann, 2003. Priming stimulation enhances the depressant effect of low-frequency repetitive transcranial magnetic stimulation. J. Neurosci., 23: 10867-10872. PMID: 14645480

Kim, Y.H., S.H. You, M.H. Ko, J.W. Park and K.H. Lee et al., 2006. Repetitive transcranial magnetic stimulation-induced corticomotor excitability and associated motor skill acquisition in chronic stroke. Stroke, 37: 1471-1476. PMID: 16675743

Knops, A., H.C. Nuerk, R. Sparing, H. Foltys and K. Willmes, 2006. On the functional role of human parietal cortex in number processing: How gender mediates the impact of a 'virtual lesion' induced by rTMS. Neuropsychologia, 44: 2270-2283. PMID: 16828812

Kobayashi, M. and A. Pascual-Leone, 2003. Transcranial magnetic stimulation in neurology. Lancet Neurol., 2, 145-156. PMID: 12849236

Kossev, A., S. Siggelkow, H. Kapels, R. Dengler and J.D. Rollnik, 2001. Crossed effects of muscle vibration on motor evoked potentials. Clin. Neurophysiol., 112: 453-456. PMID: 11222966

Kujirai, T., M.D. Caramia, J.C. Rothwell, B.L. Day and P.D. Thompson et al., 1993. Corticocortical inhibition in human motor cortex. J. Physiol., 471: 501-519. PMID: 8120818

Lisanby, S.H., D. Gutman, B. Luber, C. Schroeder and H.A. Sackeim, 2001. Sham TMS: Intracerebral measurement of the induced electrical field and the induction of motor-evoked potentials. Biol. Psychiatry, 49: 460-463. PMID: 11274658

Lisanby, S.H., L.H. Kinnunen and M.J. Crupain, 2002. Applications of TMS to therapy in psychiatry. J. Clin. Neurophysiol., 19: 344-360. PMID: 12436089 
Loo, C.K., J.L. Taylor, S.C. Gandevia, B.N. McDarmont and P.B. Mitchell et al., 2000. Transcranial Magnetic Stimulation (TMS) in controlled treatment studies: Are some "sham" forms active? Biol. Psychiatry, 47: 325-331. PMID: 10686267

Machado, S., J. Bittencourt, D. Minc, C.E. Portella and B. Velasques et al., 2008. Therapeutic applications of repetitive transcranial magnetic stimulation in clinical neurorehabilitation. Funct. Neurol., 23: 113-122. PMID: 19152730

Machado, S., M. Cunha, B. Velasques, D. Minc and S. Teixeira et al., 2010. Sensorimotor integration: basic concepts, abnormalities related to movement disorders and sensorimotor training-induced cortical reorganization. Rev. Neurol., 51: 427-436. PMID: 20859923

Maeda, F., J.P. Keenan, J.M. Tormos, H. Topka and A. Pascual-Leone, 2000. Interindividual variability of the modulatory effects of repetitive transcranial magnetic stimulation on cortical excitability. Exp. Brain Res., 133: 425-430. PMID: 10985677

Mansur, C.G., F. Fregni, P.S. Boggio, M. Riberto and J. Gallucci-Neto et al., 2005. A sham stimulation controlled trial of rTMS of the unaffected hemisphere in stroke patients. Neurology, 64: 1802-1804. PMID: 15911819

Marzi, C.A., C. Miniussi, A. Maravita, L. Bertolasi and G. Zanette et al., 1998. Transcranial magnetic stimulation selectively impairs interhemi- spheric transfer of visuo-motor information in humans. Exp. Brain Res., 118: 435-438. PMID: 9497152

Miller, E.K. and J.D. Cohen, 2001. An integrative theory of prefrontal cortex function. Annu. Rev. Neurosci., 24: 167-202. PMID: 11283309

Modugno, N., Y. Nakamura, C.D. MacKinnon, S.R. Filipovic and S. Bestmann et al., 2001. Motor cortex excitability following short trains of repetitive magnetic stimuli. Exp. Brain Res., 140: 453-459. PMID: 11685398

Muellbacher, W., U. Ziemann J. Wissel, N. Dang and M. Kofler et al., 2002. Early consolidation in human primary motor cortex. Nature, 415: 640644. PMID: 11807497

Murase, N., J.C. Rothwell, R. Kaji, R. Urushihara and K. Nakamura et al., 2005. Subthreshold lowfrequency repetitive transcranial magnetic stimulation over the premotor cortex modulates writer's cramp. Brain, 128: 104-115. PMID: 15483042

Murillo-Rodriguez, E., O. Arias-Carrion, K. SanguinoRodriguez, M. Gonzalez-Arias and R. Haro, 2009. Mechanisms of sleep-wake cycle modulation. CNS
Neurol. Disord. Drug Targets, 8: 245-253. PMID: 19689306

Nahas, Z., M. Lomarev, D.R. Roberts, A. Shastri and J.P. Lorberbaum et al., 2001. Unilateral left prefrontal Transcranial Magnetic Stimulation (TMS) produces intensity-dependent bilateral effects as measured by interleaved BOLD fMRI. Biol. Psychiatry, 50: 712-720. PMID: 11704079

O'Reardon, J.P., A.D. Peshek, P.R. Romero and P. Cristancho, 2006. Neuromodulation and Transcranial Magnetic Stimulation (TMS): A 21st Century paradigm for therapeutics in psychiatry. Psychiatry, 3: 30-40. PMID: 21103148

O'Reardon J.P., H.B. Solvason, P.G. Janicak, S. Sampson and K.E. Isenberg et al., 2007. Efficacy and safety of transcranial magnetic stimulation in the acute treatment of major depression: A multisite randomized controlled trial. Biol. Psychiatry, 62: 1208-1216. PMID: 17573044

Pascual-Leone, A. and J.M. Tormos-Munoz, 2008. Transcranial magnetic stimulation: The foundation and potential of modulating specific neuronal networks. Rev. Neurol., 46: S3-10. PMID: 18302119

Pascual-Leone, A., J. Valls-Sole, E.M. Wassermann and M. Hallett, 1994. Responses to rapid-rate transcranial magnetic stimulation of the human motor cortex. Brain, 117: 847-858. PMID: 7922470

Pascual-Leone, A., V. Walsh and J. Rothwell, 2000. Transcranial magnetic stimulation in cognitive neuroscience--virtual lesion, chronometry and functional connectivity. Curr. Opin. Neurobiol., 10: 232-237. PMID: 10753803

Quartarone, A., H.R. Siebner and J.C. Rothwell, 2006. Task-specific hand dystonia: Can too much plasticity be bad for you? Trends Neurosci., 29: 192-199. PMID: 16519953

Quartarone, A., V. Rizzo, S. Bagnato, F. Morgante and A. Sant'Angelo et al., 2005. Homeostatic-like plasticity of the primary motor hand area is impaired in focal hand dystonia. Brain, 128: 19431950. PMID: 15872016

Rosenkranz, K. and J.C. Rothwell, 2004. The effect of sensory input and attention on the sensorimotor organization of the hand area of the human motor cortex. J. Physiol., 561: 307-320. PMID: 15388776

Rosenkranz, K., A. Williamon, K. Butler, C. Cordivari and A.J. Lees et al., 2005. Pathophysiological differences between musician's dystonia and writer's cramp. Brain, 128: 918-931. PMID: 15677703 
Rossi, S., M. Ferro, M. Cincotta, M. Ulivelli and S. Bartalini et al., 2007. A Real Electro-Magnetic Placebo (REMP) device for Sham Transcranial Magnetic Stimulation (TMS). Clin. Neurophysiol., 118: 709-716. PMID: 17188568

Rossini, P.M., A.T. Barker, A. Berardelli, M.D. Caramia and G. Caruso et al., 1994. Non-invasive electrical and magnetic stimulation of the brain, spinal cord and roots: Basic principles and procedures for routine clinical application. Report of an IFCN committee. Electroencephalogr. Clin. Neurophysiol., 91: 79-92. PMID: 7519144

Rossini, P.M., L. Rossini and F. Ferreri, 2010. Brainbehavior relations: Transcranial magnetic stimulation: A review. IEEE Eng. Med. Biol. Mag., 29: 84-95. PMID: 20176526

Sandrini, M., C. Umiltà and E. Rusconi, 2011. The use of transcranial magnetic stimulation in cognitive neuroscience: A new synthesis of methodological issues. Neurosci. Biobehav. Rev., 35: 516-536. PMID: 20599555

Sandrini, M., P.M. Rossini and C. Miniussi, 2004. The differential involvement of inferior parietal lobule in number comparison: An rTMS study. Neuropsychologia, 42: 1902-1909. PMID: 15381020

Schonfeldt-Lecuona, C., L. Cardenas-Morales, R.W. Freudenmann, T. Kammer and U. Herwig, 2010. Transcranial magnetic stimulation in depression-lessons from the multicentre trials. Restor Neurol. Neurosci., 28: 569-576. PMID: 20714079

Shah, D.B., L. Weaver and J.P. O'Reardon, 2008. Transcranial magnetic stimulation: a device intended for the psychiatrist's office, but what is its future clinical role? Expert Rev. Med. Devices, 5: 559-66. PMID: 18803466

Siebner, H.R., J.M. Tormos, A.O. Ceballos-Baumann, C. Auer and M.D. Catala et al., 1999. Low frequency repetitive transcranial magnetic stimulation of the motor cortex in writer's cramp. Neurology, 52: 529-537. PMID: 10025782

Speer, A.M., T.A. Kimbrell, E.M. Wassermann, D.J. Repella and M.W. Willis et al., 2000. Oppositive effects of high and low frequency rTMS on regional brain activity in depressed patients. Biol. Psychiatry, 48: 1133-1141. PMID: 11137053

Takeuchi, N., T. Chuma, Y. Matsuo, I. Watanabe and K. Ikoma, 2005. Repetitive transcranial magnetic stimulation of contralesional primary motor cortex improves hand function after stroke. Stroke, 36: 2681-2686. PMID: 16254224
Talelli, P., R.J. Greenwood and J.C. Rothwell 2007. Exploring theta burst stimulation as an intervention to improve motor recovery in chronic stroke. Clin. Neurophysiol., 118: 333-342. PMID: 17166765

Thickbroom, G.W., 2007. Transcranial magnetic stimulation and synaptic plasticity: experimental framework and human models. Exp. Brain Res., 180: 583-593. PMID: 17562028

Touge, T., W. Gerschlager, P. Brown and J.C. Rothwell, 2001. Are the after-effects of lowfrequency rTMS on motor cortex excitability due to changes in the efficacy of cortical synapses. Clin. Neurophysiol., 112: 2138-2145. PMID: 11682353

Tsubokawa, T., Y. Katayama, T. Yamamoto, T. Hirayama and S. Koyama, 1993. Chronic motor cortex stimulation in patients with thalamic pain. J. Neurosurg., 78: 393-401. PMID: 8433140

Tyc, F. and A. Boyadjian, 2006. Cortical plasticity and motor activity studied with transcranial magnetic stimulation. Rev. Neurosci., 17: 469-95. PMID: 17180875

Walsh, V. and M, Rushworth, 1999. A primer of magnetic stimulation as a tool for neuropsychology. Neuropsychologia, 37: 125-135. PMID: 10080370

Werhahn, K.J., J. Mortensen, R.W. Van Boven, K.E. Zeuner and L.G. Cohen, 2002. Enhanced tactile spatial acuity and cortical processing during acute hand deafferentation. Nat. Neurosci., 5: 936-938. PMID: 12219095

Williams, J.A., M. Imamura and F. Fregni, 2009. Updates on the use of non-invasive brain stimulation in physical and rehabilitation medicine. J. Rehabil. Med., 41: 305-311. PMID: 19363560

Wu, T., M. Sommer, F. Tergau and W. Paulus, 2000. Lasting influence of repetitive transcranial magnetic stimulation on intracortical excitability in human subjects. Neurosci. Lett., 287: 37-40. PMID: 10841985

Ziemann, U, 2004. TMS induced plasticity in human cortex. Rev. Neurosci., 15: 253-266. PMID: 15526550 